\title{
Genotoxic therapy stimulates error-prone DNA repair in dormant hepatocellular cancer stem cells
}

\author{
SHIMPEI NISHIKAWA ${ }^{1,2}$, HIDESHI ISHII ${ }^{1}$, NAOTSUGU HARAGUCHI ${ }^{1}$, YOSHIHIRO KANO $^{1,2}$, \\ TAKAHITO FUKUSUMI $^{1,2}$, KATSUYA OHTA ${ }^{1,2}$, MIYUKI OZAKI ${ }^{1,2}$, DAISUKE SAKAI $^{1}$, \\ TAROH SATOH $^{1}$, HIROAKI NAGANO $^{2}$, YUICHIRO DOKI ${ }^{2}$ and MASAKI MORI ${ }^{2}$ \\ Departments of ${ }^{1}$ Frontier Science for Cancer and Chemotherapy, and ${ }^{2}$ Gastroenterological Surgery, \\ Osaka University, Graduate School of Medicine, Suita, Osaka 565-0871, Japan
}

Received October 20, 2011; Accepted January 6, 2012

DOI: $10.3892 /$ etm.2012.522

\begin{abstract}
Previous studies have described distinct dormant and proliferating populations of cancer stem cells in hepatocellular carcinoma. The CD13 protein is involved in the scavenging of reactive oxygen species through the glutathione reductase pathway and is associated with resistance to chemotherapy. Whereas CD13- proliferating cancer stem cells are sensitive to chemotherapy, $\mathrm{CD}_{13}{ }^{+}$dormant cancer stem cells are associated with the development of resistance to chemotherapy. CD13 ${ }^{+}$cells in hypoxic areas of the tumour survive chemotherapy, leading to subsequent disease relapse and metastasis. Whether $\mathrm{CD} 13^{+}$dormant cells simply resume proliferation following therapy or whether they also acquire greater malignant potential, remains unknown. The mechanisms involved also remain unclear. In the present study, we investigated the repair of DNA damage in CD13 ${ }^{+}$ dormant and $\mathrm{CD}^{-} 3^{-}$proliferating cells. Total RNA was extracted from tissues, and quantitative real-time polymerase chain reaction (PCR) was performed for specific genes and GAPDH following PCR. Products were then subjected to a temperature gradient of $55-95^{\circ} \mathrm{C}$ with continuous fluorescence monitoring to generate a melting curve. Cells were incubated with primary antibodies, washed twice, incubated with fluorescent-labelled secondary antibodies for $30 \mathrm{~min}$ on ice and analyzed by flow cytometry. The results revealed that the repair of DNA damage in $\mathrm{CD}_{13}{ }^{+}$dormant cells occurs predominantly through non-homologous end-joining, a repair process that is error-prone, whereas CD13- proliferating cells
\end{abstract}

Correspondence to: Professor Hideshi Ishii, Department of Frontier Science for Cancer and Chemotherapy, Osaka University, Graduate School of Medicine, Suita, Yamadaoka 2-2, Osaka 565-0871, Japan

E-mail: hishii@gesurg.med.osaka-u.ac.jp

Dr Masaki Mori, Department of Gastroenterological Surgery, Osaka University, Graduate School of Medicine, Suita, Yamadaoka 2-2, Osaka 565-0871, Japan

E-mail: mmori@gesurg.med.osaka-u.ac.jp

Key words: repair, cancer stem cells, chemo-resistance primarily utilise high-fidelity homologous recombination for DNA repair. These data indicate that not only is dormancy a protective mechanism for cancer stem cells to survive therapy, but it also enhances the generation and accumulation of mutations following DNA damage. Therefore, the $\mathrm{CD} 13^{+}$ dormant cancer stem cells must be eradicated fully to achieve complete remission of cancer.

\section{Introduction}

Double-stranded breaks (DSBs) in genomic DNA are potentially fatal for all cells, and damage-induced repair mechanisms are necessary in order to ensure cell survival (1). There are at least two fundamentally different repair mechanisms: Homologous recombination (HR) and non-homologous end-joining (NHEJ) (1). The activation of cell-cycle checkpoints by DNA damage leads to mitotic arrest until the damage is repaired. Once the damage is repaired, cells will re-enter mitosis regardless of whether the repair process introduced errors in the DNA sequences $(2,3)$. DNA damage that is too severe to be successfully repaired instead triggers programmed cell death $(4,5)$. Thus, DNA damage and the fidelity of the repair process are critical in determining whether cells survive with a normal phenotype, or become malignant with an abberant one.

Cancer stem cells (CSCs) are the major cause of resistance to therapy, disease recurrence and metastasis to other organs (6-8). As with the stem cells that underlie homeostasis in normal tissues, such as skin, intestine and the haematopoietic system (9), CSCs may be either dormant (dCSCs) or actively dividing (aCSCs). In hepatocellular carcinoma, $\mathrm{CD}^{+} 3^{+} \mathrm{dCSCs}$ residing in hypoxic areas of the tumour in the liver margin are capable of surviving chemotherapy or radiotherapy (10). The incidence of DSBs in $\mathrm{CD}_{13}{ }^{+} \mathrm{dCSC}$ is low following chemotherapy, most likely due to the scavenging of reactive oxygen species (ROS) by CD13 (aminopeptidase N) (10). This is in contrast to $\mathrm{CD} 13^{-\mathrm{CD}} 90^{+/-} \mathrm{dCSC}$, which undergo ROS-induced cell death following chemotherapy (11). Significantly, chemotherapy results in a shift in the CSC population from aCSCs to dCSCs, with dormancy acting as a refuge for the survival of malignant cells. CD13 is critical in suppressing ROS-dependent cell death during the epithelial-mesenchymal 


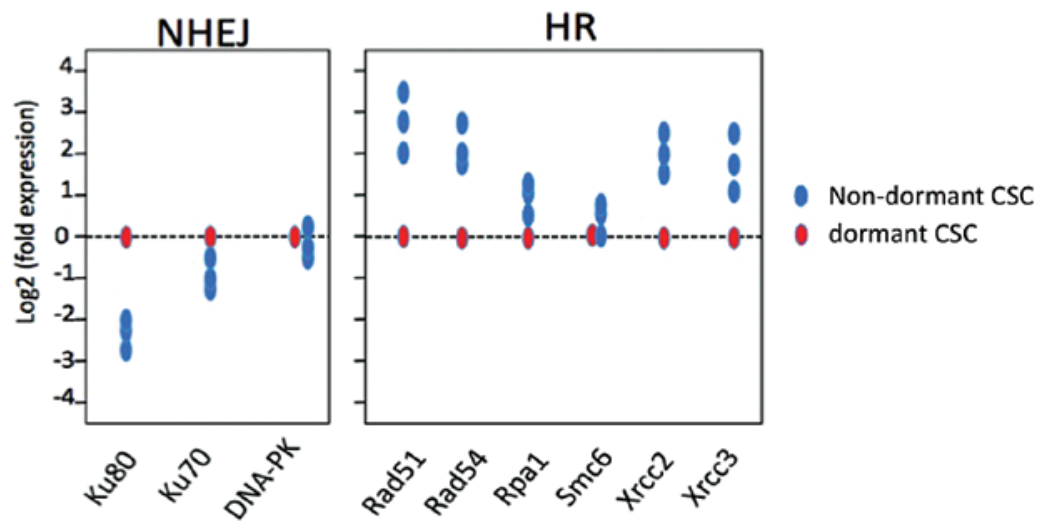

Figure 1. Quantitative analysis of mRNAs encoding proteins involved in HR and NHEJ. A total of $24 \mathrm{~h}$ after exposure to 7 Gy radiation, cells were separated by FACS into pools of dormant $\mathrm{CD} 13^{+} \mathrm{CSC}$ and actively dividing (non-dormant) $\mathrm{CD} 13^{-} \mathrm{CSC}$. The RNAs were extracted and subjected to quantitative analysis of PCR by utilising specific primers. For HR, Rad51, Rad54, Rpa, Smc6, Xrcc2 and Xrcc3 were evaluated, whereas Ku80, Ku70 and Prkdc were assessed for NHEJ. HR, homologous recombination; NHEJ, non-homologous end-joining; CSCs, cancer stem cells; PCR, polymerase chain reaction.

transition of CSCs during metastasis (12). Exposure to the CD13-specific inhibitor, ubenimex, results in considerable eradication of malignant cells in vivo, indicating the likely benefit of the combination of conventional chemotherapy with the CD13-specific inhibitor.

In this study, we show that DSBs in dCSCs result predominantly in the activation of DNA repair via the NHEJ pathway, whereas DNA repair by HR is the predominant response in aCSCs. Based on these data, we propose that dCSCs should be the major target of therapy in order to completely eradicate the malignant cell population, although hibernation therapy (the induction to dormancy) may be a suitable rationale dependent on the medical condition of patients.

\section{Materials and methods}

Cell culture. Cell lines were maintained in Dulbecco's modified Eagle's medium (DMEM; Nacalai Tesque, Kyoto, Japan) supplemented with $10 \%$ foetal bovine serum (FBS) in a humidified incubator at $37^{\circ} \mathrm{C}$ in $5 \% \mathrm{CO}_{2}$. All plasmids were purchased from Addgene (Cambridge, MA, USA).

$R N A$. Total RNA was extracted from tissues using TRIzol reagent (Invitrogen, Tokyo, Japan), and cDNA was synthesised using SuperScript III RT kits (Invitrogen). Quantitative realtime polymerase chain reaction (qRT-PCR) for specific genes and GAPDH was performed using a LightCycler TaqMan Master kit with specific primers provided by the manufacturer (Roche Diagnostics, Tokyo, Japan). The amplification protocol consisted of 35 cycles of denaturation at $95^{\circ} \mathrm{C}$ for $10 \mathrm{sec}$ and annealing and elongation at $60^{\circ} \mathrm{C}$ for $30 \mathrm{sec}$. The products were then subjected to a temperature gradient of $55-95^{\circ} \mathrm{C}$ with continuous fluorescence monitoring to generate a melting curve. mRNA expression levels were normalised in comparison to GAPDH mRNA.

Protein. Cell surface phenotypes were characterised by flow cytometry on a FACSAria (BD, Tokyo, Japan). Cells were incubated with primary antibodies in cold PBS containing $0.1 \%$ FBS for $1 \mathrm{~h}$ on ice, washed twice, incubated with fluo- rescent-labelled secondary antibodies for $30 \mathrm{~min}$ on ice and analyzed by flow cytometry.

Statistical analysis. For continuous variables, the results were expressed as the means \pm standard error. The correlation between the gene expression level and cell count was analysed by Chi-square and Wilcoxon rank tests. All data were analysed using JMP software (SAS Institute, Cary, NC, USA). A value of $\mathrm{p}<0.05$ was considered to indicate a statistically significant difference.

\section{Results}

In hepatocellular carcinoma, CD13 is expressed in CSCs (10) and is involved in the scavenging of ROS via the glutathione reductase pathway. CD90 has been proposed as a marker of stem cells, and its expression is correlated with tumourigenicity in mouse in vivo tumour models and the clinical outcome in human patients (13). In hepatocellular carcinoma, the $\mathrm{CD}_{13}{ }^{+} \mathrm{CD} 90^{-}$cell population is predominantly dormant, $\mathrm{CD}_{13}{ }^{+} \mathrm{CD} 90^{+}$cells are predominantly in the $\mathrm{S}$ phase and $\mathrm{CD}^{-} 3^{-} \mathrm{CD} 90^{+}$cells are in the G2/M phase (10). Following exposure to genotoxic insults, such as chemotherapy or radiation therapy, the proportions of these cell populations shift; CD13- cells become less common, whereas the dormant $\mathrm{CD}_{13}{ }^{+}$population increases. We were interested in the mechanism of DNA repair in the various CSC populations; therefore, we used fluorescence-activated cell sorting (FACS) to separate dormant $\mathrm{CSCs}\left(\mathrm{CD} 13^{+}\right)$from non-dormant CSCs $\left(\mathrm{CD}^{-} 3^{-}\right)$and analysed the expression of proteins involved in the various DNA repair pathways. Rad51, Rad54, Rpa, Smc6, $\mathrm{Xrcc} 2$ and $\mathrm{Xrcc} 3$ are involved in HR, whereas Ku80, Ku70 and Prkdc are indicative of NHEJ. We found that the expression ratio of NHEJ-associated proteins to HR-associated proteins increased in dormant CSCs, indicating that DSBs are repaired predominantly through NHEJ in these cells. DNA repair by NHEJ is also the dominant mechanism in quiescent hematopoietic stem cells (16). Conversely, in non-dormant CSCs, HR-associated repair proteins increased relative to NHEJ-associated proteins (Figs. 1-3). 


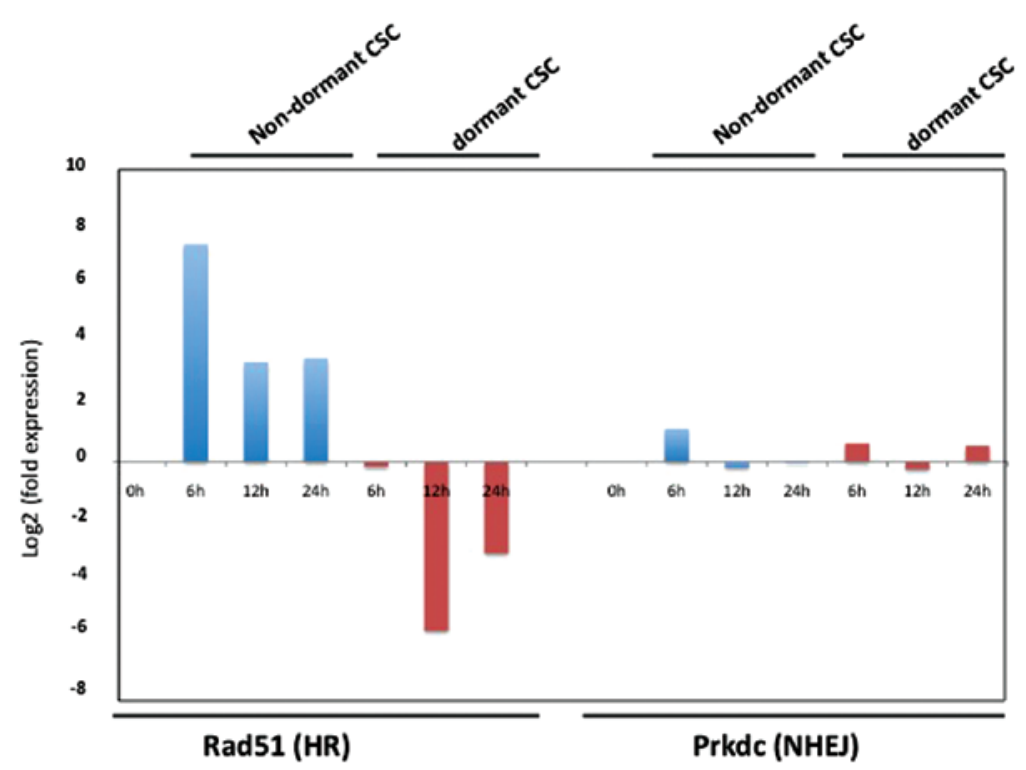

Figure 2. The time course experiment of expression of NHEJ and HR proteins. Cells were separated by surface markers, dormant CSCs (CD13 $\left.{ }^{+}\right)$and non-dormant CSCs (CD13) at the indicated time following 7 Gy radiation. cDNAs were synthesised and subjected to PCR. The expression of Rad51 (HR) and Prkdc (NHEJ) were evaluated by qRT-PCR. HR, homologous recombination; NHEJ, non-homologous end-joining; CSCs, cancer stem cells; qRT-PCR, quantitative real-time polymerase chain reaction.

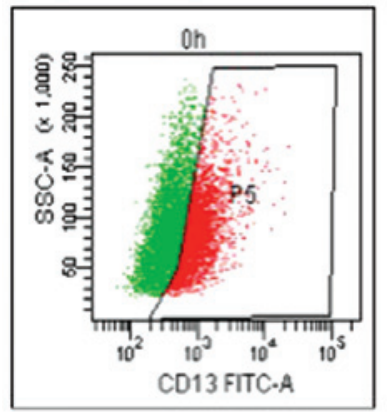

$27.2 \%$

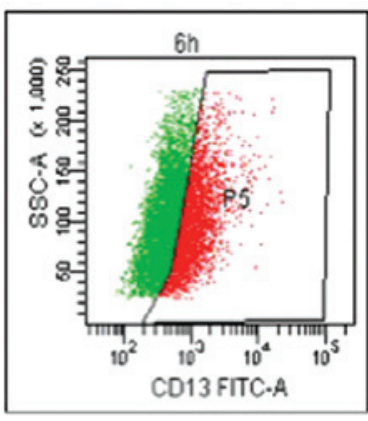

$28.3 \%$

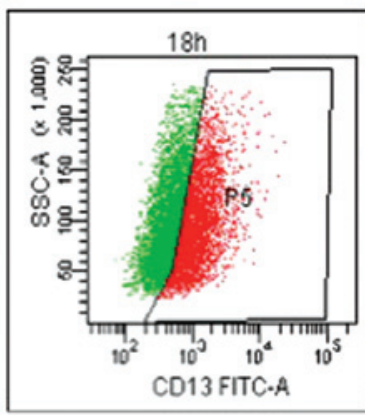

$32.7 \%$

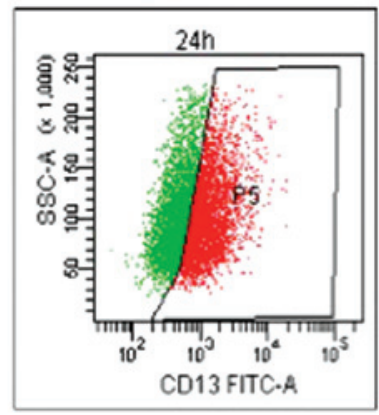

$39.9 \%$

Figure 3. FACS analysis of $\mathrm{CD}^{+} 3^{+}$dormant CSCs following exposure to radiation (7 Gy). Following exposure to radiation, $\mathrm{CD} 13^{+}$cells are shown as a percentage. FACS, fluorescence-activated cell sorting; CSCs, cancer stem cells.

\section{Discussion}

In proliferating CSCs, either in whole tissues or tissue culture, exposure to genotoxic insults, such as chemotherapy and radiation therapy, causes DNA damage during the $\mathrm{S}$ phase of the cell cycle, followed by apoptosis of tumour cells (4). In a fraction of the actively dividing tumour cells that survive therapy, the DNA damage is repaired by HR, consistent with the data shown in this study. DNA repair by HR is typically a high-fidelity, low-error mechanism. By contrast, dormant cells are more likely to utilise NHEJ for DNA repair. NHEJ may occur at any stage of the cell cycle but has only a limited requirement for sequence homology and, importantly, is more prone to error than repair by HR (1). Thus, NHEJ is associated with the misrepair of DSBs, and may result in chromosomal deletions, insertions or translocations and subsequent genomic instability (14). These genomic alterations may lead to the inactivation of tumour suppressor genes or activation of oncogenes, which lead to the development of primary and metastatic tumours. In our study, we found that DNA repair by NHEJ is characteristic of dormant CSCs. Due to the mutagenic effects of NHEJ, dormant CSCs that survive chemoradiation therapy may acquire new mutations and become more likely to cause tumour recurrence and/or metastasis (15). It is therefore critical to eradicate dCSCs in order to improve patient survival.

Quiescence is widely accepted to be an essential protective mechanism for stem cells that minimises the endogenous stress caused by cellular respiration and DNA replication in normal cells (16). However, it is also important in tumour evolution after therapy, since quiescent cells that survive therapy are likely to have acquired mutations during DNA repair by NHEJ (8). Thus, genotoxic therapies, such as chemoradiation therapy, that suppress genotoxic effects are a double-edged sword, removing primary tumour bulk, but also contributing to tumour recurrence after therapy. 


\section{References}

1. Sancar A, Lindsey-Boltz LA, Unsal-Kacmaz K and Linn S Molecular mechanisms of mammalian DNA repair and the DNA damage checkpoints. Annu Rev Biochem 73: 39-85, 2004.

2. Bartek J and Lukas J: Chk1 and Chk2 kinases in checkpoint control and cancer. Cancer Cell 3: 421-429, 2003.

3. Bartek J, Lukas C and Lukas J: Checking on DNA damage in Sphase. Nat Rev Mol Cell Biol 5: 792-804, 2004.

4. Lowe SW and Lin AW: Apoptosis in cancer. Carcinogenesis 21: 485-495, 2000

5. Kunkel TA: DNA replication fidelity. J Biol Chem 279: 16895-16898, 2004

6. Reya T, Morrison SJ, Clarke MF and Weissman IL: Stem cells, cancer, and cancer stem cells. Nature 414: 105-111, 2001.

7. Visvader JE and Lindeman GJ: Cancer stem cells in solid tumours: accumulating evidence and unresolved questions. Nat Rev Cancer 10: 755-768, 2008

8. Dewi DL, Ishii H, Kano Y, Nishikawa S, Haraguchi N, Sakai D, Satoh T, Doki Y and Mori M: Cancer stem cell theory in gastrointestinal malignancies: recent progress and upcoming challenges. J Gastroenterol 46: 1145-1157, 2011.

9. $\mathrm{Li} \mathrm{L}$ and Clevers $\mathrm{H}$ : Coexistence of quiescent and active adult stem cells in mammals. Science 327: 542-545, 2010.

10. Haraguchi N, Ishii H, Mimori K, Tanaka F, Ohkuma M, Kim HM, Akita H, Takiuchi D, Hatano H, Nagano H, Barnard G, Doki Y and Mori M: CD13 is a therapeutic target in human liver cancer stem cells. J Clin Invest 120: 3326-33239, 2010.
11. Haraguchi N, Ishii H, Nagano H, Doki Y and Mori M: The future prospects and subject of the liver cancer stem cells study for the clinical application. Gastroenterology: Feb 23, 2011 (Epub ahead of print).

12. Kim HM, Haraguchi N, Ishii H, Ohkuma M, Okano M, Mimori K, Eguchi H, Yamamoto H, Nagano H, Sekimoto M, Doki $Y$ and Mori M: Increased CD13 expression reduces reactive oxygen species, promoting survival of liver cancer stem cells via an epithelial-mesenchymal transition-like phenomenon. Ann Surg Oncol: Aug 31, 2011 (Epub ahead of print).

13. Yang ZF, Ho DW, Ng MN, Lau CK, Yu WC, Ngai P, Chu PW, Lam CT, Poon RT and Fan ST: Significance of CD90 cancer stem cells in human liver cancer. Cancer Cell 13: 153-166, 2008.

14. Weinstock DM, Richardson CA, Elliott B and Jasin M: Modeling oncogenic translocations: distinct roles for double-strand break repair pathways in translocation formation in mammalian cells. DNA Repair (Amst) 5: 1065-1074, 2006.

15. Ishii H, Iwatsuki M, Ieta $K$, Ohta D, Haraguchi N, Mimori K and Mori M: Cancer stem cells and chemoradiation resistance. Cancer Sci 99: 1871-1877, 2008.

16. Mohrin M, Bourke E, Alexander D, Warr MR, Barry-Holson K, Le Beau MM, Morrison CG and Passegué E: Hematopoietic stem cell quiescence promotes error-prone dna repair and mutagenesis. Cell Stem Cell 7: 174-185, 2010. 